\title{
To Know Your Own Strength: Overriding Natural Force Attenuation
}

\author{
Nicole L. Valles and Kyle B. Reed, Member, IEEE
}

\begin{abstract}
The human sensorimotor system is thought to attenuate the perception of self-generated forces, which causes people to generate a larger force so the perception of their exerted force matches their intention [1]. The purpose of this study is to understand the nature of force attenuation, to determine if the natural tendency to underestimate self-generated forces can be retrained, and to ascertain if humans can learn to more accurately recreate forces. To test this hypothesis, forces were applied to one hand and each participant was asked to recreate those forces on his/her other hand. The back and forth force exchange generated the expected force escalation in individuals. Participants were then given feedback about the accuracy of their recreated forces during a training phase. Before training, no participants had their average force in the correct range, but $86 \%$ of participants had their average force in the correct range when tested the following day. The participants also increased the consistency of their force recreation after training.
\end{abstract}

Index Terms-perception and psychophysics, force attenuation, habituation, performance, learning, active and passive touch

\section{INTRODUCTION}

$\mathrm{W}$ HEN guiding an individual, a specific position is dictated by applying a force or a specific force is dictated by imposing a position, but force and position cannot both be imposed simultaneously. When teaching someone a physical skill, such as flying a helicopter or providing physical therapy, the act of the expert/therapist physically guiding the novice/patient limits either the perception of interaction force or the perception of motion. In addition, passively having a force applied is perceived differently than an actively applied force [2]. Thus, humans cannot accurately perceive the full interaction another person had with an environment. During the training of physical skills, being able to more fully experience both the force and motion involved in the interaction would be highly beneficial.

The goal of this continuing work is to understand how force and motion can be accurately perceived and recreated so an individual can learn and experience the interactions another individual had with an environment. Our previous study [3] examined how an individual can recreate a motion applied to one hand with the other hand. The study presented here examines the related ability to bimanually perceive and recreate a force. Past studies have demonstrated a natural tendency to attenuate self-generated forces, which leads to an escalation in force [1][4]. The specific question posed here is: can humans be made aware of the force escalation effect and, therefore, be able to accurately replicate the experienced stimuli? In other words, once shown how their natural tendency detrimentally affects their performance, can people learn to more accurately recreate the force?

- N. Valles and K.B.Reed are with the Department of Mechanical Engineering, University of South Florida.

E-mail: kylereed@usf.edu

\section{BACKGROUND}

Several studies have suggested that internal models in the central nervous system predict the outcomes of actions [5][6][2]. These predicted outcomes are proposed to attenuate the perception when the action occurs. In the simplest form, the predicted response is subtracted from the actual response, which reduces the perception intensity [2]. Walsh et al. [4] show that the attenuation has a constant offset and another component that varies with the level of force. These two factors explain why external actions are not completely eliminated. These studies applied a force to one finger and the participants were asked to recreate that force on the same or different hand. An alternative explanation for the altered recreation of force is habituation, which is a decrease in the response strength that occurs through repeated presentations of a stimulus.

The consequences of decreasing the response from actively applied forces are that "self-generated forces are perceived as weaker than externally generated forces of the same magnitude", which leads to larger forces when individuals are asked to recreate a force applied to another finger [1]. This reduced perception of self-generated forces also explains why it is difficult to tickle oneself [7]. The underlying neural mechanism for this effect is not currently known and humans are generally unaware of the effect [5].

Experience and training have been shown to overcome incorrect perceptions of external objects. One example is the size-weight illusion. People generally perceive larger objects to weigh less than smaller objects of the same mass. Flanagan et al. [8] showed that the expectation of weight can be altered by training individuals with objects that follow an inverse relationship between size and weight. They also showed that the sensorimotor system rapidly learned the inverse mapping. Our current study examines if individuals can similarly learn to overcome the natural attenuation of self-generated forces and accurately recreate a force in a bimanual task. 
This is the author's version of an article that has been published in this journal. Changes were made to this version by the publisher prior to publication. The final version of record is available at http://dx.doi.org/10.1109/TOH.2013.55

Humans are able to coordinate the actions between their limbs during bimanual tasks [9], such as while turning a steering wheel [10] and simultaneously drawing circles with both hands [11], but generally have difficulty using two hands to perform uncorrelated tasks [12]. Reinkensmeyer et al. [13] show that an individual holding a pencil between two fingers on different hands will apply slightly more force on one hand to accelerate the pencil and then switch and apply slightly more force with the other hand to stop the motion. It has also been demonstrated that motions generated simultaneously with both limbs can help an individual's ability to perform similar unimanual tasks [14].

Previous studies have examined how bimanual motions can allow an individual to recreate a motion while circumventing the ambiguities of task and guidance forces applied to the same hand. McAmis and Reed [3] examined motions recreated by moving the hands in the same direction compared to mirror motions; the performance was similar, but certain motions favored certain coupling modes. They also examined bimanual guidance in which one hand received guidance forces and the other received task forces. Powell and O'Malley [15][16] compared several guidance methods to allow an individual to learn from an expert. Their results showed that using a joystick to guide one of the user's hands and allowing the user to recreate that motion with the other hand was feasible and had a relatively low mental workload. Both of these studies examined recreating motions; the study presented here examines how an individual can accurately recreate a perceived force.

\section{Methods}

The hypothesis tested in these experiments is that people can learn to correctly perceive the forces applied passively and also learn to actively recreate those forces accurately. To examine this ability, we studied participants' ability to recreate a force applied on one hand with their other hand.

\subsection{Device Setup}

The experimental setup simultaneously provided a force to the user's left hand and measured the user's response force from their right hand (Fig. 1). The force was provided using a DC motor with a load cell fixed to the end of a 5 inch lightweight lever. The lever pushed down onto the users left indexed finger, which rested in a form-fitting plastic pad. No muscle force was required, so this configuration allowed the left index finger to perceive the force passively.

The motor torque and load cell were externally calibrated using a scale accurate to $001 \mathrm{~N}$. Since there was little motion involved and no transmission friction, the motor control was open loop and was validated in each trial using the load cell. To measure the applied force from the user's right hand, a hinged pad rested over a second load cell that was calibrated the same as the first load cell. The participant used their right index finger to generate the response forces on the right side load cell. Wrist padding was provided, however, all participants opted not to use it, presumably since this setup was similar to a computer mouse that is typically used without any support.

The system was programmed using $\mathrm{C}++$ in Microsoft Visual Studio ${ }^{\circledR}$ running on Windows $\mathrm{XP}^{\circledR}$. The motor was controlled via a voltage controlled current source op amp configuration with an analog voltage generated from a Phidget ${ }^{\circledR}$ Inc. PhidgetAnalog. The two load cells were connected via a Phidget ${ }^{\circledR}$ Inc. PhidgetInterfaceKit. The system ran at $100 \mathrm{~Hz}$, which is sufficient given the minimal dynamics involved in this setup.

\subsection{Procedure}

The participants were seated in front of the setup and asked to place their index fingers on the respective finger pads. The fingers were facing opposite directions to minimize the resulting motions and to ensure the forces were perceived as desired. The left finger was passive since the force was applied to it. The right finger actively generated the force by pushing down on the pad.

Once in position, we explained that they would feel a force applied on their left index finger and they were to recreate that force using their right index finger. They were given a short period of time to ask questions and become comfortable with the setup. Questions relating to the specific study hypotheses were deferred to the end of the experiment on the second day, at which point all questions were answered.

The experiment consisted of the following parts, described in more detail below, in this order:

1) Pre-test of force recreation (10 trials)

2) Bimanual force escalation (2 trials of 6 rounds)

3) Training force recreation (25-50 trials)

4) Post-test of force recreation (10 trials)

5) One day followup test of force recreation (10 trials)

The experiment took no more than 30 minutes on the first day and less than 10 minutes on the second day.

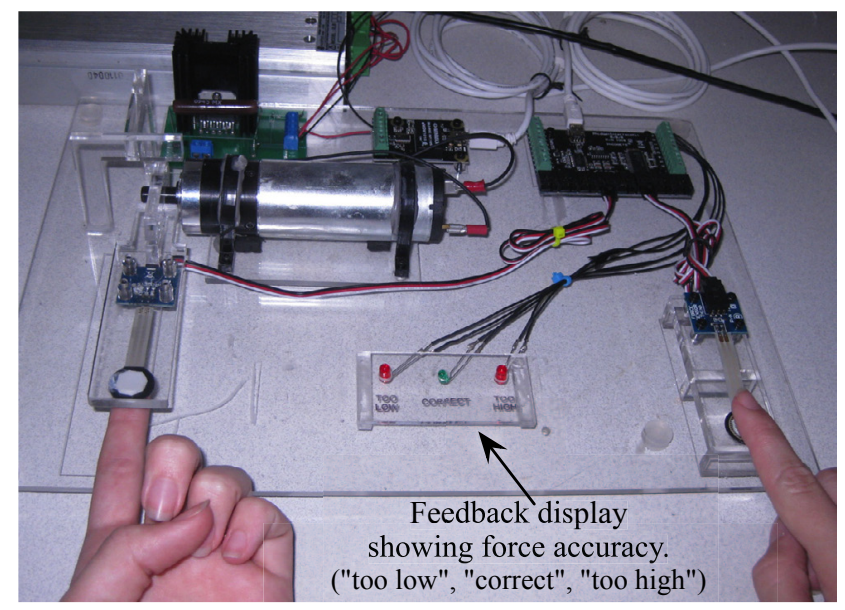

Fig. 1. The experimental setup. Participants simultaneously received a passive input force to their left index finger from the motor and actively responded with an isometric force from their right index finger. 
This is the author's version of an article that has been published in this journal. Changes were made to this version by the publisher prior to publication. The final version of record is available at http://dx.doi.org/10.1109/TOH.2013.55

\subsubsection{Testing Force Recreation}

To test the participants' natural force perception and recreation, we measured their response to several specific forces. The participants were provided a force to their left index finger and asked to replicate that force with their right index finger. The recorded response force was an average of 2 seconds collected 3 seconds after the applied force started. Each test consisted of the following ten forces applied in a random order: $1,1.5,2,2.5,3,3.5,4,4.5,5,5.5 \mathrm{~N}$. This testing procedure was done three times throughout this study: at the start, after training, and one day later.

\subsubsection{Bimanual Force Escalation}

Participants were provided with an initial force of $2 \mathrm{~N}$ to their left index finger and were told to replicate the applied force with their right index finger. They were instructed that if the force they felt with their left finger changed, they should accordingly adjust their responding force. In other words, they should always strive to keep the force received equal to the force applied. The input force was applied continuously to their left hand for 5 seconds. The response force was measured during the last 1 second. The applied force was then changed to the average of the measured force from the current force at a rate of $5 \mathrm{~N} / \mathrm{sec}$. Although the participants were unaware of the method to determine the new force, the system was set up as a feedback loop. The force applied to the user was actually the same force that the user applied. This experiment stopped when the subject applied more than $8 \mathrm{~N}$ or if they performed more than six rounds; an escalation in force was expected. The experiment was run twice for each participant. This part of the experiment served as a baseline and as a demonstration to the participants about the nature of their force perception.

\subsubsection{Training Force Recreation}

The first part of the training consisted of informing the participants of the true nature of the system. They were told that the changes they felt in motor strength during the last set of trials were actually mimicking their force responses. This was explained to them to clearly demonstrate their general inability to accurately recreate a force, thus encouraging them to try harder in the next training phase. Since they were told about the effect at this point, we did not further test them using the force escalation task as they would then have prior knowledge that would have skewed the results. We instead relied on the quantitative measure described in Section 3.2.1.

The second part of the training allowed participants to receive feedback about their recreated force. An input force would be displayed and they could respond as they had been. However, during training, three LEDs (Fig. 1) would indicate how accurately they recreated the force. When a response was within $\pm 10 \%$, the green LED would turn on, which indicated they recreated the force correctly. If they were above or below this range, the "too hight" or "too low" LED would turn on, respectively. The accurate range was defined as falling within the just noticeable difference (JND) of force perception, which Allin et al. [17] determined as $\pm 10 \%$. We do not expect that the force could be recreated more accurately than the JND for force perception. For training, participants had a minimum of 25 individual learning trials that consisted of five back-to-back trials of the following forces: $1,2,3,4$, and $5 \mathrm{~N}$. A short break was given before moving to the next block of five. Participants were allowed to repeat each force level once if they desired for a total of 50 individual learning trials. The order of presentation was randomized.

\subsection{Participants}

The study included seven participants; four males and three females. Their mean age was 24 with a range from 21 to 32 years. All participants were right handed and had no known impairments that would affect this study. This study was approved by the University of South Florida IRB and all participants signed an approved IRB consent form.

\section{Results}

\subsection{Force Escalation}

The participants generally recreated a larger force than was originally applied to them. As can be seen in Fig. 2, the effect was so pronounced that 11 out of the 14 escalation trials reached a force greater than $8 \mathrm{~N}$ in six or less rounds, at which point the trial was terminated. This escalation is expected because this pre-experiment was a single-person version of the tit-for-tat experiment [1] in which one or two people alternately recreated the force applied to them. Based on the theory of sensory attenuation, the applied force and the perceived force are not the same [18]; thus,

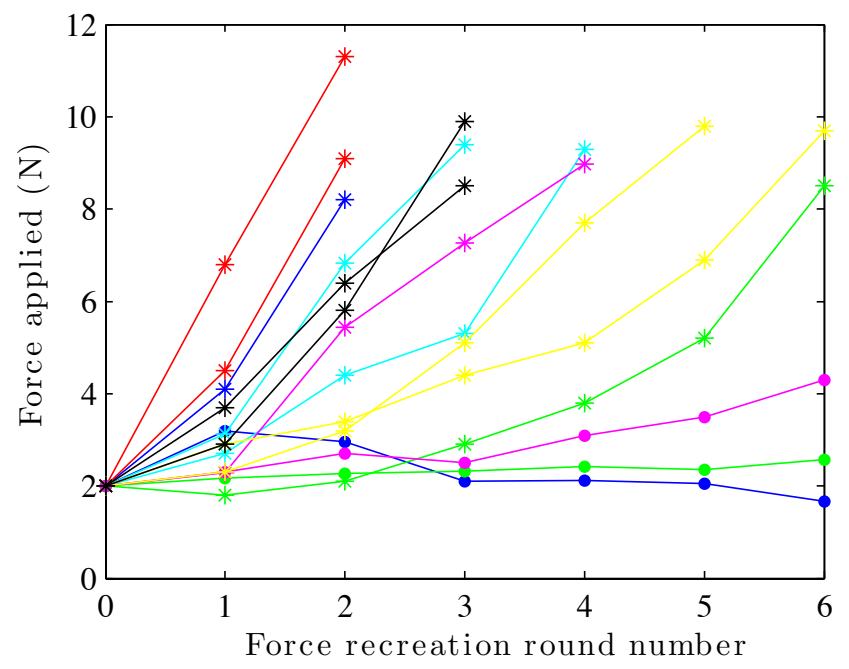

Fig. 2. The escalation for each of the two rounds for the seven participants. The escalation test was terminated when the force applied exceeded $8 \mathrm{~N}$ or they had reached the sixth round. Force values from trials that did not escalate to the $8 \mathrm{~N}$ cutoff value are marked by $a \bullet$; the rest are indicated by $a^{*}$. Each participant s two trials are the same color. 
This is the author's version of an article that has been published in this journal. Changes were made to this version by the publisher prior to publication. The final version of record is available at http://dx.doi.org/10.1109/TOH.2013.55

the individuals applied a larger force to make the force they perceived match the actual force applied to them, which caused force escalation in most instances.

In some instances, however, the participants did not escalate. The trials marked by $\bullet '$ in Fig. 2 indicate the three trials that were terminated due to reaching the sixth round. To understand why these trials did not escalate, we compared the changes in applied force in each round to the JND for force perception, which is $10 \%$ [17]. Each time the force was recreated during the non-escalating trials, the recreated force increased less than $10 \%$ of the previously applied force in 12 out of the 18 rounds (67\% of the rounds). Since the force change was less than the JND, the participants likely did not perceive that the force changed, thus they did not significantly change their recreated force and did not escalate. It is also possible that they accurately perceived the force, but did not accurately recreate it. In contrast, out of the 11 escalation trials that did reach the $8 \mathrm{~N}$ cutoff, 39 out of the 40 rounds increased by more than the $10 \%$ JND, thus they likely did perceive the change in force, which resulted in the expected force escalation.

\subsection{Recreating the Force}

Training enabled the participants to more accurately recreate the force on average. The participants were tested at the start of the experiment, after training, and one day later. The force applied to the left hand (input) was compared to the corresponding recreated force generated with the right hand (output). Fig. 3 shows histograms of the percentage of force recreated for the three periods.

The group force recreation distributions were shown to not fit a normal distribution using the Chi-Square Goodness of Fit test for normality. Thus, we used the non-parametric Kruskal-Wallis one-way analysis of variance test with percentage force recreation as the independent variable and each of the three testing periods as factors. The testing periods are statistically significantly different $\left(\chi^{2}(2,207)=64.8, p<.0001\right)$. Tukey's honestly significant difference test with an alpha of 0.05 shows that all three factors are statistically significantly different. Although the results demonstrate that the effects of training mostly persisted, there was a statistically significant decrease in their ability after one day, but the effect was small (+10 percentage points) compared to the change from the pre-test ( -39 percentage points).

Participants improved their ability to recreate a correct force after training. We define a correct trial as being within $10 \%$ of the target level, which is the JND for force perception [17]. During the pre-test, participants were only correct $11 \%$ of the time. After the participants became aware of the effects of natural attenuation and could practice with feedback about their applied forces, they were able to generate a force within the JND $46 \%$ of the time. They retained most of their improved ability after one day when they were correct $40 \%$ of the time. The persistence of the learned effect suggests that individuals can learn to overcome the escalation effect. (a) Pre-test

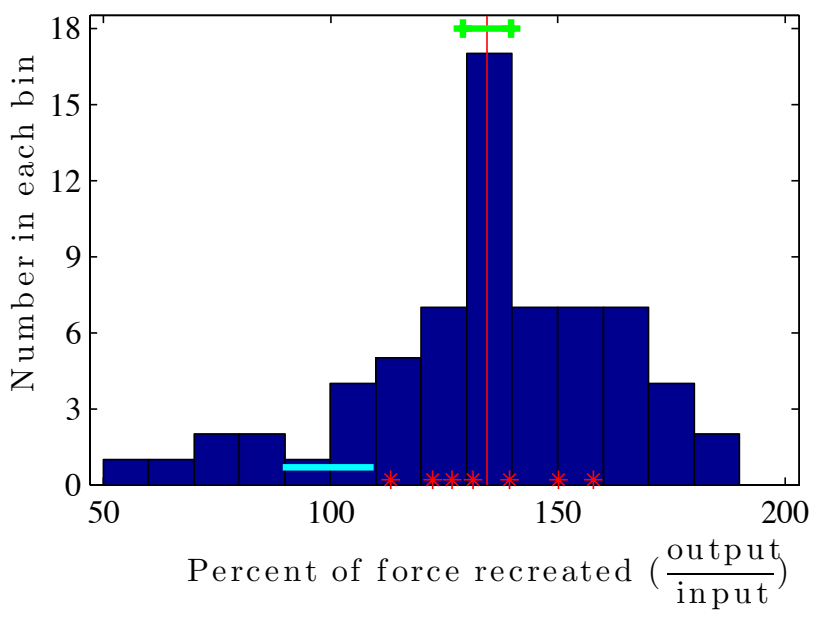

(b) Post-training test

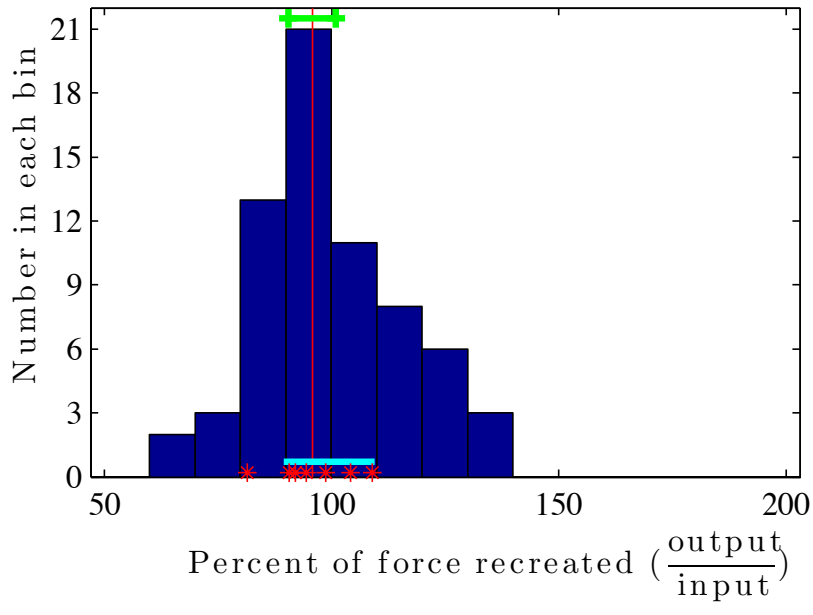

(c) One day followup test

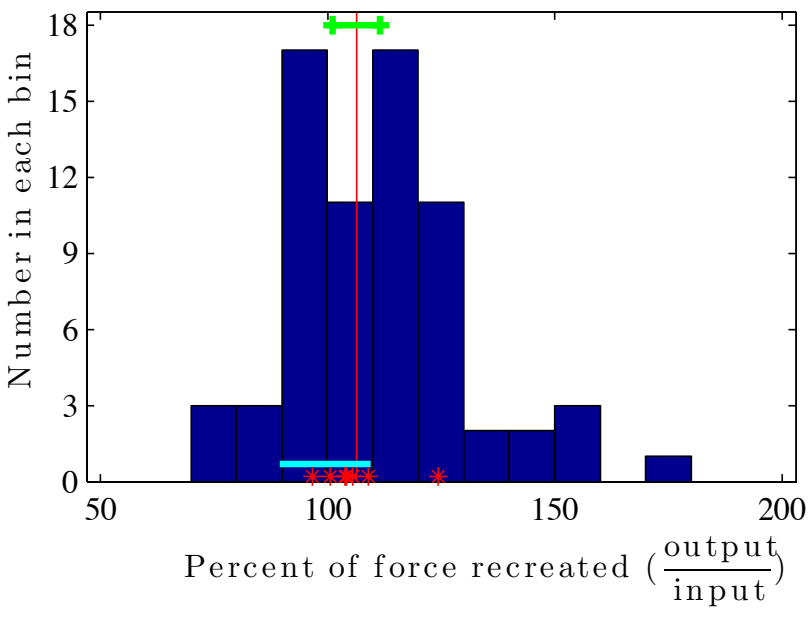

\begin{tabular}{l} 
Each bin represents 10 percentage points \\
\hline Average for all subjects \\
95\% confidence interval \\
Range for a correct trial $( \pm 10 \%)$ \\
Each participant's average
\end{tabular}

Fig. 3. Percentage of force recreated during the testing. Perfect recreation of the force is $100 \%$. 
This is the author's version of an article that has been published in this journal. Changes were made to this version by the publisher prior to publication. The final version of record is available at http://dx.doi.org/10.1109/TOH.2013.55

Although the individual trials were only right a little less than half the time, 6 of the 7 participants averaged within the $10 \%$ JND after training and one day later, whereas none of the participants had an average that was correct before training. The red *' in Fig. 3 shows the average for each individual.

The consistency of the reproduced forces also increased with training as demonstrated by the decreased standard deviation. Since the group data was not normally distributed, the standard deviation was examined by shifting all of an individual's trials so that the average of all individuals was the same. It is expected that large numbers of individual trials on this task would follow a normal distribution, but the different individual means skewed the overall distribution. A Chi-Square Goodness of Fit test for normality showed that the null hypothesis of a normal distribution could not be rejected for the shifted data for all three test periods. This assessment of consistency is based on the percentage change, but a recent study demonstrates that the attenuation also has a constant absolute offset [4]. Thus, the standard deviation was also calculated using the difference of the applied and recreated forces; the trend is similar. All of these comparisons are shown in Table 1.

There was not a significant correlation between the results of the force recreation and force escalation tasks. In other words, the participants that recreated higher forces did not tend to escalate faster.

\section{Discussion AND Future WORK}

In summary, this study demonstrates that training can help individuals to more consistently reproduce more accurate forces. The learned ability appears to be persistent, but has only been tested in a one day followup.

The force escalation effect can be compensated for or inhibited in several ways. Shergill et al. [1] showed that the generated forces were more similar to the input force when participants used a joystick to generate the output force compared to when they directly generated the force with their finger. Walsh et al. [4] showed that self-generating the target force with the other hand can reduce, and actually reverse, the attenuation effect. Another method is to show the individuals the effect and train them to compensate for the predictive mechanisms, as demonstrated in this paper. Although there are many instances where using a joystick to bypass this mechanism is acceptable, such as cooperative teleoperation, there are many scenarios where this is impractical, such as during the interaction between a physical therapist and a patient. Teaching physical

TABLE 1

Changes in Ability to Recreate Force

\begin{tabular}{|c|c|c|c|c|}
\hline Test time & $\begin{array}{c}10 \% \text { JND } \\
\text { (\% correct) }\end{array}$ & $\begin{array}{c}\text { participants } \\
\text { correct } \\
\text { on average }\end{array}$ & $\begin{array}{c}\text { std. dev. } \\
\text { based on \% } \\
\text { (aligned average) }\end{array}$ & $\begin{array}{c}\text { std. dev. based } \\
\text { on force } \\
\text { difference (N) }\end{array}$ \\
\hline Pre-test & $11 \%$ & $0 \%$ & 31 & 1.01 \\
\hline Post-test & $46 \%$ & $86 \%$ & 22 & 0.63 \\
\hline One day & $40 \%$ & $86 \%$ & 17 & 0.48 \\
\hline
\end{tabular}

therapists to generate the desired amount of force may allow them to more accurately provide the therapy that is needed.

The specific pathways for attenuation are not known. In some ways, compensating for attenuation is similar to consciously blocking a natural polysynaptic re ex action [19]. Whereas re exive actions occur along the spinal cord, the attenuation is likely occurring at higher levels of the brain. The attenuation of afferent signals arising from one's own actions (reafference) has been shown to occur when all or a subset of skin, muscle, and central signals were available [4], thus the attenuation is likely occurring on the combined estimate of force.

We used the force perception JND to classify correct responses, however, the JND likely does not account for all the error in a force recreation task. A related study demonstrated that an individual can generate the same forces on contralateral elbow exor muscles and the JND for matching force generation between limbs was similar to unimanual weight perception [20]. In that case, the individual is actively generating and matching the force between both arms at the same time, thus the individual is comparing a similar, possibly attenuated, reafferent signal from both sides. In force recreation tasks, one side has an external force applied to a passive finger resulting in an afferent signal; the other side is generating a force, which results in an attenuated reafferent signal. Some force recreation studies sequentially display the target force and then ask the participant to match the force, so a simultaneous comparison of the forces was not available, only a stored version of the perception. These two versions of force recreation show similar results, which suggests that at least one of the mechanisms for comparison is different than force matching tasks. Furthermore, the high variability of force recreation resulted in less than half the individual trials in this study being correct, yet the average forces from most participants was within the JND. These results suggest that JND alone cannot account for all of the error affecting the force recreation tasks and another source of error is likely affecting the recreation task. The large variability could also explain why some trials did not demonstrate the expected escalation - at the extreme, the recreation error could have been larger than the force attenuation, so the two effects could have cancelled out in some trials.

The variability was high, but the individuals became more consistent after training. This improvement is likely due to additional practice with recreating the force, which is not a common daily task. The standard deviation also decreased the next day without any additional practice, which could be explained by motor consolidation [21]. However, the average recreated force level increased, so the absolute level of force was not maintained. From this study, it is not clear whether the passive perception or the active recreation of the force was affected. Changing either one would allow the forces to be recreated more accurately, but in either case, the increased consistency suggests that they learned to recreate the forces more accurately.

Handedness was not considered here, but the literature suggests handedness is only important for positional 
This is the author's version of an article that has been published in this journal. Changes were made to this version by the publisher prior to publication. The final version of record is available at http://dx.doi.org/10.1109/TOH.2013.55

awareness and cooperative bimanual tasks, not the force matching tasks performed here. One study suggests that the sensorimotor system is asymmetric with a proprioceptive advantage for the nonpreferred arm [22]. A force matching study showed that there was no statistically significant difference between switching the input and output limbs. In bimanual tasks, the motions are shown to specialize to different aspects of task performance [13][23]. The symmetric perception/recreation of forces combined with the asymmetry of the proprioceptive system is potentially beneficial for bimanual guidance [15][3][16], which is a method that portrays the guidance forces to one hand and the other hand recreates the motion and feels the task relevant forces; the guidance and task forces should be felt equivalently and not attenuated while performing the motions with both hands. In addition, sensory selection suggests that only information from one hand is used at a time to perceive the interaction in bimanual tasks [24], thus the side interacting with the environment should be able to combine the motions and task-relevant forces into a single perception separate from the guidance forces.

The results from this study indicate that training and being made aware of the effects of force attenuation lead to more accurate force recreation. In further studies using this paradigm, the effects of simply being told about the effect and the training could be separated by using false information during the training session. The testing would observe the effects of only being told about escalation and also the effects of being trained with consistently incorrect forces.

\section{ACKNOWLEDGMENT}

This material is based upon work supported by the National Science Foundation under Grant No. IIS-1319802.

\section{REFERENCES}

[1] S. S. Shergill, P. M. Bays, C. D. Frith, and D. M. Wolpert, "Two eyes for an eye: The neuroscience of force escalation," Science, vol. 301, p. 187, 2003

[2] D. Wolpert, Z. Ghahramani, and M. Jordan, "An internal model for sensorimotor integration," Science, vol. 269, no. 5232, p. 1880, 1995.

[3] S. McAmis and K. B. Reed, "Simultaneous perception of forces and motions using bimanual interactions," IEEE Trans. on Haptics, vol. 5, no. 3, pp. 220-230, 2012.

[4] L. D. Walsh, J. L. Taylor, and S. C. Gandevia, "Overestimation of force during matching of externally generated forces," The Journal of physiology, vol. 589, no. 3, pp. 547-557, 2011.

[5] S. Blakemore, D. Wolpert, and C. Frith, "Abnormalities in the awareness of action," Trends in Cognitive Sciences, vol. 6, no. 6, pp. 237-242, 2002.

[6] D. Wolpert, "Computational approaches to motor control," Trends in cognitive sciences, vol. 1, no. 6, pp. 209-216, 1997.

[7] S. Blakemore, D. Wolpert, and C. Frith, "Central cancellation of selfproduced tickle sensation," Nature neuroscience, vol. 1, pp. 635-640, 1998.

[8] J. Flanagan, J. Bittner, R. Johansson et al., "Experience can change distinct size-weight priors engaged in lifting objects and judging their weights," Current Biology, vol. 18, no. 22, pp. 1742-1747, 2008.

[9] H. G. Malabet, R. A. Robles, and K. B. Reed, "Symmetric motions for bimanual rehabilitation," in Proc. IEEE/RSJ Int Intelligent Robots and Systems (IROS) Conf, 2010, pp. 5133-5138.
[10] M. Johnson, H. Van der Loos, C. Burgar, P. Shor, and L. Leifer, "Experimental results using force-feedback cueing in robot-assisted stroke therapy," IEEE Trans. on Neural Sys. and Rehabilitation Engr., vol. 13, pp. 335-348, 2005.

[11] R. Carson, J. Thomas, J. Summers, M. Walters, and A. Semjen, "The dynamics of bimanual circle drawing," The Quarterly J. of Experimental Psychology: Section A, vol. 50, pp. 664-683, 1997.

[12] F. Mechsner, D. Kerzel, G. Knoblich, and W. Prinz, "Perceptual basis of bimanual coordination," Nature, vol. 414, no. 6859, pp. 69-72, 2001.

[13] D. Reinkensmeyer, P. Lum, and S. Lehman, "Human control of a simple two-hand grasp," Biological cybernetics, vol. 67, no. 6, pp. 553-564, 1992.

[14] J. Burgess, R. Bareither, and J. Patton, "Single limb performance following contralateral bimanual limb training," IEEE Trans. on Neural Systems and Rehabilitation Engr., vol. 15, pp. 347-355, 2007.

[15] D. Powell and M. K. O'Malley, "Efficacy of shared-control guidance paradigms for robot-mediated training," in Proc. IEEE World Haptics Conf., 2011, pp. 427-432.

[16] D. Powell and M. K. O'Malley, "The task-dependent efficacy of shared-control haptic guidance paradigms," IEEE Trans. on Haptics, vol. 5, no. 3, pp. 208-219, 2012.

[17] S. Allin, Y. Matsuoka, and R. Klatzky, "Measuring just noticeable differences for haptic force feedback: implications for rehabilitation," in Proc. Haptic Interfaces for Virtual Environment and Teleoperator Systems, 2002, pp. 299-302.

[18] S. J. Blakemore, D. Wolpert, and C. Frith, "Why can't you tickle yourself?" Neuroreport, vol. 11, no. 11, pp. R11-R16, 2000.

[19] P. Crago, J. Houk, and Z. Hasan, "Regulatory actions of human stretch re ex," Journal of Neurophysiology, vol. 39, no. 5, pp. 925935, 1976.

[20] L. A. Jones et al., "Matching forces: constant errors and differential thresholds," Perception, vol. 18, no. 5, pp. 681-687, 1989.

[21] R. Shadmehr and H. H. Holcomb, "Neural correlates of motor memory consolidation," Science, vol. 277, no. 5327, pp. 821-825, 1997. [Online]. Available: http://www.sciencemag.org/content/277/ 5327/821.abstract

[22] D. J. Goble and S. H. Brown, "Upper limb asymmetries in the perception of proprioceptively determined dynamic position sense." J. of Experimental Psychology: Human Perception and Performance, vol. 36, no. 3, p. 768, 2010.

[23] J. Wang and R. L. Sainburg, "The dominant and nondominant arms are specialized for stabilizing different features of task performance," Experimental Brain Research, vol. 178, no. 4, pp. 565-570, 2007.

[24] V. Squeri, A. Sciutti, M. Gori, L. Masia, G. Sandini, and J. Konczak, "Two hands, one perception: how bimanual haptic information is combined by the brain," J. of Neurophys, vol. 107, no. 2, pp. 544$550,2012$.

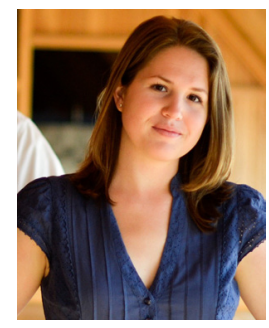

Nicole Valles received her BS in mechanical engineering from the University of South Florida, Tampa Florida. She is currently living and working in Seattle, WA as a Commissioning Project Manager and is actively pursuing her professional engineering license. She is an alumni member of Theta Tau. Her interests include haptics and human-machine interaction.

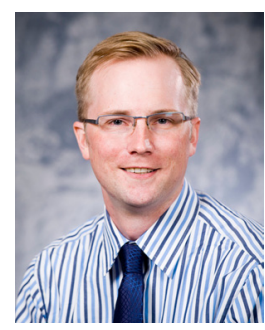

Kyle B. Reed is an assistant professor of Mechanical Engineering at the University of South Florida. He received the BS degree from the University of Tennessee and the MS and PhD degrees from Northwestern University, all in mechanical engineering. $\mathrm{He}$ was a postdoctoral fellow in the Laboratory for Computational Sensing and Robotics at the Johns Hopkins University. His interests include haptics, human-machine interaction, rehabilitation, medical robotics, and engineering education. $\mathrm{He}$ is an IEEE member. 